\title{
Anthocyanin-Rich Blueberry Extract Ameliorates the Behavioral Deficits of MPTP-Induced Mouse Model of Parkinson's Disease via Anti-Oxidative Mechanisms
}

\author{
Feng Qian ${ }^{1 *}$, Mengmeng Wang2* , Jianggang Wang' ${ }^{2}$ Chengbiao Lu ${ }^{1,2 \#}$ \\ ${ }^{1}$ Laboratory of Neuronal Network and Brain Diseases Modulation, School of Medicine, Yangtze University, \\ Jingzhou, China \\ ${ }^{2}$ The Key Laboratory of the Brain Research of Henan Province, Department of Pathology, Xinxiang Medical University, \\ Xinxiang, China \\ Email: "johnlu9000@hotmail.com
}

How to cite this paper: Qian, F.., Wang, M.M., Wang, J.G. and Lu, C.B. (2019) Anthocyanin-Rich Blueberry Extract Ameliorates the Behavioral Deficits of MPTP-Induced Mouse Model of Parkinson's Disease via Anti-Oxidative Mechanisms Yangtze Medicine, 3, 72-78.

https://doi.org/10.4236/ym.2019.31008

Received: September 2, 2018

Accepted: March 19, 2019

Published: March 22, 2019

Copyright $\odot 2019$ by author(s) and Scientific Research Publishing Inc. This work is licensed under the Creative Commons Attribution International License (CC BY 4.0).

http://creativecommons.org/licenses/by/4.0/

Open Access

\begin{abstract}
The pathological process of Parkinson's disease (PD) involves oxidative stress, mitochondrial dysfunction and dopaminergic neuronal loss. Recent studies have suggested that the fruits rich in anthocyanins (ANC) were neuroprotective, and then could reduce the risk of $\mathrm{PD}$. This study was designed to investigate the effects of the ANC rich blueberry extracts (BBE) on the behavior and the oxidative stress in the mouse model of $\mathrm{PD}$ induced by 1-methyl-4-phenyl-1,2,3,6-tetrahydropyridine (MPTP). After 3 weeks of BBE administration, we monitored the behavioral alterations and measured the level of dopamine (DA), enzymatic tyrosine hydroxylase (TH), non-enzymatic malonaldehyde (MDA), enzymatic superoxide dismutase (SOD) and glutathione peroxidase (GSH-PX) in the brain and serum. Our results demonstrated that the behavioral impairment, the increased MDA level and the decreased SOD and GSH-PX activities in the MPTP-induced PD mice, were significantly attenuated by BBE administration $(\mathrm{p}<0.05)$. This study suggested that anthocyanin-rich BBE should have neuroprotective effects through the anti-oxidative mechanisms.
\end{abstract}

\section{Keywords}

Blueberry Extract, Parkinson's Disease, Oxidative Stress

${ }^{\star}$ These two authors contributed equally to this article. 


\section{Introduction}

Parkinson's disease (PD) is a progressive neurodegenerative disease, characterized by bradykinesia, rigidity, resting tremor and unsteadiness due to the loss of dopaminergic cells and the reduced dopamine level in the midbrain substantia nigra [1]. Tyrosine hydroxylase (TH), an enzyme responsible for catalyzing the conversion of the L-tyrosine to L-3,4-dihydroxyphenylalanine (L-DOPA), the precursor of dopamine, is decreased in PD [2]. Mitochondrial failure and oxidative stress appear to be two major contributors to nigral neuronal death in PD. Deficiency in Complex I subunits of the respiratory chain in the PD patients may result in the over-production of reactive oxygen species (ROS) and the promotion of the deposition of fibril forms of a-synuclein [3] [4]. 1-methyl-4-phenyl-1,2,3,6-tetrahydropyridine (MPTP), a mitochondrial complex I inhibitor, decreased the levels of $\mathrm{TH}$, depleted dopamine in nigral-striatal area and mimics symptoms of PD in the mouse model [5]. It was also reported that chronic exposure of environmental pollutants such as rotenone, another mitochondrial complex I inhibitor which was used as an insecticide and pesticide, increased the risk of $\mathrm{PD}[6]$.

The main treatment of PD is the application of levodopa [7]. Alternatively, the plant extracts attenuated the degeneration of dopamine neurons in PD model [8]. The blueberry extract (BBE), which contain phytochemicals including anthocyanins (ANC) and proanthocyanidins, enhanced the cognitive function in both children and older adults [9] [10]. Blueberry polyphenols also attenuated kainic acid-induced decrements in cognition in rat hippocampus [11]. Dietary supplementation with BBE improved survival of transplanted dopamine neurons [12] and alleviated neurodegeneration induced by rotenone in a cellular model [13]. These data suggested that BBE should play a beneficial role for the PD patients.

In this study, we measured behavior parameter, dopamine content, enzymatic TH activity, non-enzymatic malonaldehyde (MDA) level, and enzymatic superoxide dismutases (SOD) and glutathione peroxidase (GSH-PX) activities which are important antioxidants in nearly all cells, and investigated the effects of BBE on above indices in the MPTP induced PD model C57BL/6 mice, aimed at determining the possible role and mechanism of ANC rich BBE.

\section{Materials and Methods}

\subsection{Reagents}

MPTP and L-DOPA were purchased from Sigma Aldrich (Shanghai, China). Blueberry extract were purchased from Xi'an Qingzhi Biotech Co. Ltd (Xi'an, China) and was formulated for oral gavages. DA, TH, SOD, GSH-PX and MDA measurement kits were purchased from Nanjing Jiancheng Bioengineering Institute (Nanjing, China).

\subsection{Animal Grouping and Administration}

All animal studies were conducted in accordance with the Guide for the Care 
and Use of Laboratory Animals as adopted and promulgated by Yangtze University, Hubei, China. C57BL/6 mice, male, 6 weeks old, weighing 18 - 22 g (purchased from the Experimental Animal Center, Institute of Military Medical Sciences, Beijing, China) were randomly divided into normal control group, MPTP + saline group, MPTP + BBE and MPTP + positive drug group (9 animals in each group). For the normal control group, animals were reared under normal circumstances and received intraperitoneal injection (i.p.) of saline. For the MPTP + saline group, each animal was received daily i.p. of MPTP $30 \mathrm{mg} / \mathrm{kg}$ for 5 days and the saline by gavages for 3 weeks [5]. For the MPTP + BBE groups, each animal was received daily i.p. of MPTP $30 \mathrm{mg} / \mathrm{kg}$ for 5 days and BBE (50 $100 \mathrm{mg} / \mathrm{kg}$ ) by gavages for 3 weeks. For the positive drug group, each animal was received daily i.p. of MPTP and fed daily with levodopa and benserazide (10 $\mathrm{mg} / \mathrm{kg} /$ day). On the day 21 , behavior tests were performed. After the tests, the mice were sacrificed and the whole brain of each mouse was quickly taken by cutting the skull in the middle line. Brain tissue was weighed and added in ice-cold saline $\left(4^{\circ} \mathrm{C}\right)$ in accordance with a ratio of quality to volume $(1: 10)$. A $10 \%$ homogenate was made followed by centrifugation for $10 \mathrm{~min}$ at $1000 \mathrm{~g} / \mathrm{min}$, $4^{\circ} \mathrm{C}$. The supernatant was stored at $-20^{\circ} \mathrm{C}$ and ready for the various biochemical testing.

\subsection{Motor Coordination Measurements}

Before MPTP injection, mice were trained at several different rod rotation speeds, and motor skill performance was calculated as the mean latency to fall from the rotating rod [14]. Baseline motor coordination in the rotarod test was recorded for each mouse $24 \mathrm{~h}$ after training was completed. 21 days after BBE treatment, hypokinesia-like symptoms were assessed by a constant speed (20 RPM) rotarod test. The tests consisted of three trials (180 s) with an inter-trial interval of $30 \mathrm{~min}$. Test performance was defined as the mean of the three trials. Mice were tested starting $1.5 \mathrm{~h}$ after the last therapeutic treatment.

\subsection{ELISA Detection of the Levels of DA and TH in Mouse Brain and Serum}

The two-step double-antibody sandwich enzyme-linked immunosorbent assay (ELISA) test was performed. Standards and test samples were added onto a microplate pre-coated with antibodies specific for dopamine (DA) and tyrosine hydroxylase (TH). After removing any unbound substances, a biotin-conjugated antibody specific for DA or TH is added to the wells. After washing, avidin conjugated Horseradish Peroxidase (HRP) was added to the wells. Following a wash to remove any unbound avidin-enzyme reagent, a substrate (TMB) solution was added to the wells and color develops in proportion to the amount of DA or TH in the sample (HRP catalyzed substrate and converted to the blue product and become yellow under the action of an acid). The color development was stopped and the intensity of the color was measured (The OD value was measured under $450 \mathrm{~nm}$ wavelength. The sample content of DA or TH was calculated according 
to the standard and sample OD value).

\subsection{Measurement of SOD, GSH-PX and MDA Contents in Mouse Brain and Serum}

Mice were sacrificed and the whole brain removed, weighed and added in ice-cold saline $\left(4^{\circ} \mathrm{C}\right)$ in a mass-volume ratio of $1: 10$ to prepare a $10 \%$ homogenate. The homogenate was then centrifuged for $10 \mathrm{~min}$ at $1000 \mathrm{~g} / \mathrm{min}, 4^{\circ} \mathrm{C}$, the supernatant was preserved at $-20^{\circ} \mathrm{C}$. Measurement of MDA concentration and SOD and GSH-PX activities in mouse brain were performed according to the instruction of purchased kits from Nanjing Jiancheng Bioengineering Institute, including the MDA assay kit (2-thiobarbituric acid, TBA method), and the SOD assay kit (hydroxylamine method), GSH-PX assay kit (Colorimetric method), respectively.

\subsection{Statistical Analysis}

The statistical software, Sigmastat was used for the statistical analysis. The results were expressed as mean \pm standard deviation ( $x \pm s$ ). Statistical analysis for two groups were performed using t-test analysis or a rank test and for multiple groups using ANOVA or ANOVA on ranks. ${ }^{\star} \mathrm{p}<0.05$ indicates statistically significant.

\section{Results and Discussion}

Given the data listed in Table 1, we demonstrated that the continuous MPTP treatment for 5 days $(30 \mathrm{mg} / \mathrm{kg} /$ day) significantly reduced the ability of mice to balance on a rotating rod by measuring the mean latency to fall in rotarod test ( $\mathrm{p}$ $<0.01$ ), which meant the MPTP treatment successfully induced motor coordination impairment of the PD model mice. The mouse brain DA, TH, SOD, and GSH-PX contents in the MPTP group were significantly lower than the control group ( $\mathrm{p}<0.01$ ), while the MDA content in the MPTP group were significantly

Table 1. Effects of BBE treatment on MPTP-induced PD mice.

\begin{tabular}{ccccccc}
\hline $\begin{array}{c}\text { Mice group } \\
(\mathbf{n}=9)\end{array}$ & $\begin{array}{c}\text { Mean } \\
\text { latency } \\
\text { to fall (s) }\end{array}$ & $\begin{array}{c}\text { DA } \\
(\mathrm{U} / \mathrm{mg} \\
\text { protein) }\end{array}$ & $\begin{array}{c}\text { TH } \\
\text { (nmol/mg } \\
\text { protein) }\end{array}$ & $\begin{array}{c}\text { MDA } \\
\text { (nmol/mg } \\
\text { protein) }\end{array}$ & $\begin{array}{c}\text { SOD } \\
\text { (U/mg } \\
\text { protein) }\end{array}$ & $\begin{array}{c}\text { GSH-PX } \\
\text { (nmol/mg } \\
\text { protein) }\end{array}$ \\
\hline Control & $174.1 \pm 6.6$ & $465.1 \pm 33.2$ & $522.8 \pm 56.0$ & $2.34 \pm 0.23$ & $217.3 \pm 14.6$ & $42.1 \pm 3.5$ \\
MPTP & $62.0 \pm 7.0^{\#}$ & $273.3 \pm 13.2^{\#}$ & $215.2 \pm 29.6^{\#}$ & $3.96 \pm 0.34^{\#}$ & $182.1 \pm 17.6^{\#}$ & $24.3 \pm 3.8^{\#}$ \\
BBE 50 mg/kg & $87.6 \pm 9.5^{*}$ & $359.5 \pm 24.5^{*} 386.8 \pm 22.9^{*}$ & $2.88 \pm 0.51^{*}$ & $231.4 \pm 29.3^{*}$ & $36.0 \pm 3.2^{*}$ \\
BBE 100 mg/kg & $90.0 \pm 6.8^{*}$ & $378.0 \pm 25.5^{*} 403.9 \pm 28.4^{*} 2.61 \pm 0.49^{*}$ & $263.5 \pm 40.2^{*}$ & $35.9 \pm 4.8^{*}$ \\
Madopar & $95.0 \pm 10.3^{*}$ & $385.3 \pm 22.5^{*} 477.1 \pm 35.2^{*}$ & $2.50 \pm 0.53^{*}$ & $248.0 \pm 26.4^{*}$ & $39.2 \pm 5.8^{*}$ \\
\hline
\end{tabular}

Abbreviations and symbols: MPTP, 1-methyl-4-phenyl-1,2,3,6-tetrahydropyridine, $30 \mathrm{mg} / \mathrm{kg} / \mathrm{day}$ for 5 days; BBE, blueberry extract, 50 or $100 \mathrm{mg} / \mathrm{kg} /$ day for 21 days; Madopar, Levodopa and benserazide, 10 $\mathrm{mg} / \mathrm{kg} /$ day for 21 days; DA, dopamine; TH, tyrosine hydroxylase; MDA, malonaldehyde; SOD, superoxide dismutase; GSH-PX, glutathione peroxidase; PD, Parkinson's disease. ${ }^{*} \mathrm{p}<0.05$, compared with MPTP induced PD mice; ${ }^{*} \mathrm{p}<0.01$, compared with control mice (male C57BL/6 mice, 9 per group). 
higher than that $(\mathrm{p}<0.01)$. These data indicated that the MPTP treatment induced significant oxidative stress to the model mice and decreased the content of DA in the mouse brain which finally lead to the motor coordination impairment. In this study, we demonstrated that both low $(50 \mathrm{mg} / \mathrm{kg})$ and high $(100$ $\mathrm{mg} / \mathrm{kg}$ ) BBE treatments improved motor coordination and increased DA and TH contents in PD mice $(\mathrm{p}<0.05)$. Furthermore, as a possible mechanism of its neuroprotective effect, BBE increased SOD and GSH-PX activity and decreased MDA level in the brains of MPTP-treated mice $(\mathrm{p}<0.05)$. Compared with the group treated with Levodopa and benserazide $(10 \mathrm{mg} / \mathrm{kg})$, the $\mathrm{BBE}$ treatment achieved similar therapeutic efficacy to the induced motor coordination impairment by MPTP treatment. However, different from the previously reported data which showed that the Levodopa and benserazide treatment could significantly increase the oxidative stress [15], our results suggested the opposite effect of it. The only possibility was that the Levodopa and benserazide treatment had reversed the oxidative stress induced by MPTP treatment by their neuroprotective effects [16].

$\mathrm{TH}$ has been used as a marker for dopaminergic neurons [17]. BBE significantly increased TH contents in MPTP-treated mice $(\mathrm{p}<0.05)$, which suggested that BBE could increase the survival of dopamine neurons in PD mice. Increased oxidative stress could lead to neurodegeneration in the PD via protein and/or lipid peroxidation and disruption of mitochondrial function [18]. Our results demonstrated the anti-oxidative effect of BBE, which should contribute to its neuroprotective role in PD mice, as reported previously [19] [20].

\section{Conclusion}

In conclusion, our data provided evidence that $\mathrm{BBE}$ improved motor function in MPTP-induced PD mice via a possible mechanism of its anti-oxidative capacity to scavenge free radicals and reduced oxidative damage to the neurons. The detailed mechanism remained to be further studied.

\section{Acknowledgements}

This work was supported by The National Natural Science Foundation of China (grant numbers: 31070938, 81271422, 81771517), the Applied Basic Research Plan Key Program of Hebei Province of China (grant number: 12966119D).

\section{Conflicts of Interest}

The authors declare no conflicts of interest regarding the publication of this paper.

\section{References}

[1] Pagonabarraga, J., Kulisevsky, J., Strafella, A.P. and Krack, P. (2015) Apathy in Parkinson's Disease: Clinical Features, Neural Substrates, Diagnosis, and Treatment. Lancet Neurology, 14, 518-531. https://doi.org/10.1016/S1474-4422(15)00019-8

[2] Haavik, J. and Toska, K. (1998) Tyrosine Hydroxylase and Parkinson's Disease. 
Molecular Neurobiology, 16, 285-309. https://doi.org/10.1007/BF02741387

[3] Betarbet, R., et al. (2000) Chronic Systemic Pesticide Exposure Reproduces Features of Parkinson's Disease. Nature Neuroscience, 3, 1301-1306. https://doi.org/10.1038/81834

[4] Rochet, J.C., Hay, B.A. and Guo, M. (2012) Molecular Insights into Parkinson's Disease. Progress in Molecular Biology and Translational Science, 107, 125-188. https://doi.org/10.1016/B978-0-12-385883-2.00011-4

[5] Jackson-Lewis, V., Jakowec, M., Burke, R.E. and Przedborski, S. (1995) Time Course and Morphology of Dopaminergic Neuronal Death Caused by the Neurotoxin 1-Methyl-4-phenyl-1,2,3,6-tetrahydropyridine. Neurodegeneration, 4, 257-269. https://doi.org/10.1016/1055-8330(95)90015-2

[6] Ramkumar, M., et al. (2018) Demethoxycurcumin, a Natural Derivative of Curcumin Abrogates Rotenone-Induced Dopamine Depletion and Motor Deficits by Its Antioxidative and Anti-Inflammatory Properties in Parkinsonian Rats. Pharmacognosy Magazine, 14, 9-16. https://doi.org/10.4103/pm.pm_113_17

[7] Freitas, M.E., Ruiz-Lopez, M. and Fox, S.H. (2016) Novel Levodopa Formulations for Parkinson's Disease. CNS Drugs, 30, 1079-1095.

https://doi.org/10.1007/s40263-016-0386-8

[8] Amro, M.S., et al. (2018) The Potential Role of Herbal Products in the Treatment of Parkinson's Disease. Clinical Therapeutics, 169, e23-e33.

[9] Whyte, A.R., Schafer, G. and Williams, C.M. (2016) Cognitive Effects Following Acute Wild Blueberry Supplementation in 7- to 10-Year-Old Children. European Journal of Nutrition, 55, 2151-2162. https://doi.org/10.1007/s00394-015-1029-4

[10] Miller, M.G., Hamilton, D.A., Joseph, J.A. and Shukitt-Hale, B. (2017) Dietary Blueberry Improves Cognition among Older Adults in a Randomized, Double-Blind, Placebo-Controlled Trial. European Journal of Nutrition, 57, 1169-1180. https://doi.org/10.1007/s00394-017-1400-8

[11] Shukitt-Hale, B., et al. (2008) Blueberry Polyphenols Attenuate Kainic Acid-Induced Decrements in Cognition and Alter Inflammatory Gene Expression in Rat Hippocampus. Nutritional Neuroscience, 11, 172-182. https://doi.org/10.1179/147683008X301487

[12] McGuire, S.O., et al. (2006) Dietary Supplementation with Blueberry Extract Improves Survival of Transplanted Dopamine Neurons. Nutritional Neuroscience, $\mathbf{9}$, 251-258. https://doi.org/10.1080/10284150601086134

[13] Strathearn, K.E., et al. (2014) Neuroprotective Effects of Anthocyanin- and Proanthocyanidin-Rich Extracts in Cellular Models of Parkinsons Disease. Brain Research, 1555, 60-77. https://doi.org/10.1016/j.brainres.2014.01.047

[14] Ayton, S., et al. (2013) The Effect of Dopamine on MPTP-Induced Rotarod Disability. Neuroscience Letters, 543, 105-109. https://doi.org/10.1016/j.neulet.2013.02.066

[15] Alirezaei, M., et al. (2015) Beneficial Antioxidant Properties of Betaine against Oxidative Stress Mediated by Levodopa/Benserazide in the Brain of Rats. Journal of Physiological Sciences, 65, 243-252. https://doi.org/10.1007/s12576-015-0360-0

[16] Jami, M.S., et al. (2014) Proteome Analysis Reveals Roles of L-DOPA in Response to Oxidative Stress in Neurons. BMC Neuroscience, 15, 93.

[17] Ishikawa, S., et al. (2010) Human DJ-1-Specific Transcriptional Activation of Tyrosine Hydroxylase Gene. The Journal of Biological Chemistry, 285, 39718-39731. https://doi.org/10.1074/jbc.M110.137034

[18] Ischiropoulos, H. and Beckman, J.S. (2003) Oxidative Stress and Nitration in Neu- 
rodegeneration: Cause, Effect, or Association? Journal of Clinical Investigation, 111, 163-169. https://doi.org/10.1172/JCI200317638

[19] Vendrame, S., et al. (2013) Wild Blueberry (Vaccinium angustifolium) Consumption Improves Inflammatory Status in the Obese Zucker Rat Model of the Metabolic Syndrome. The Journal of Nutritional Biochemistry, 24, 1508-1512.

https://doi.org/10.1016/j.jnutbio.2012.12.010

[20] Giacalone, M., et al. (2011) Antioxidant and Neuroprotective Properties of Blueberry Polyphenols: A Critical Review. Nutritional Neuroscience, 14, 119-125. https://doi.org/10.1179/1476830511Y.0000000007 